\title{
SMART HOME METER PROFILER WITH LOAD AUTHENTICATION, SHOCK PROTECTION, FAULT PROOF AND RESTRICTED DEMAND MANAGEMENT
}

\author{
Shashikumar K. ${ }^{*}$, Venkataseshaiah C. ${ }^{2}$, Sim Kok Swee ${ }^{2}$ \\ ${ }^{1}$ Faculty of Engineering, Multimedia University, Persiaran Multimedia, 63100 Cyberjaya, Malaysia \\ ${ }^{2}$ Faculty of Engineering and Technology, Multimedia University, 75450 Melaka, Malaysia
}

(Received: December 2018 / Revised: May 2019 / Accepted: August 2019)

\begin{abstract}
In this paper, we have designed and installed a shock or fault protection system for a single-phase home electrical system using Circuit Breaker (CB) devices available on the market, bearing in mind that certain product areas do not provide shock protection to consumers. Our novel Smart Meter Circuit Breaker (SMCB) is designed to achieve a fail-proof shock protection system architecture that could be incorporated into existing Smart Meters (SM) within a Device Level Load Meter (DLLM).

We also illustrate how a colonized and alienated appliance Authentication Outlet (AO) system could be used advantageously for a shock or fault protection system and for our novel Restricted Demand Management system with the help of a distinctive load signature alienated appliance AO. Our Restricted Demand Management system could be used together with the Scheduler Demand Management system already on the market.

Finally, this paper shows how our SMCB for an alienated appliance AO detection system for a single-phase home system was simulated, built and tested. The proposed home layout and the Restricted Demand Management System are simple to program and implement and help to reduce energy bills as only particular appliances are allowed to operate at certain times during the day and night. The system also restricts some hazard risk appliances from being used at other outlets. Our proposed system is completely hazard, fault and shockproof for a single phase home electrical system.
\end{abstract}

Keywords: Authentication outlet; Circuit breaker; Demand management; Protection; Smart meters

\section{INTRODUCTION}

The power protection systems in single-phase system homes are not fully hazard proof, and none of the products on the market can ensure full protection from utility hazards. This is apparent from the fact that there are still incidents of fatal injuries and death due to electrical shock or of homes being destroyed by fire due to electrical hazards.

To understand the effect of electric shock, we need to understand the Red Blood Cell (RBC) membrane of human tissue first, as explained in an experimental study by Fricke and Mose in 1925. They discovered the capacitancy and resistivity of the RBC membrane at $50 \mathrm{~Hz}$, with an

\footnotetext{
*Corresponding author's email: shashikumar@mmu.edu.my, Tel. +6016-6176173, Fax. +603-83183029 Permalink/DOI: https://dx.doi.org/10.14716/ijtech.v10i7.3250
} 
alternating current at $0.81 \mu \mathrm{F} / \mathrm{cm} 2$ and $200 \Omega \mathrm{cm}$. Further analysis by Alrawi et al. (2010) shows a human body circuit of a capacitor and resistor connected in parallel and series, as shown in Figure 1. With this data, we simulated and discovered that a leading $30 \mathrm{~mA}$ alternating current is required for an average-sized human body to feel an electrical shock. Figure 1 shows our newly classified electrical hazard table for understanding the areas covered by existing protection devices and which electrical hazard classification types do not protect you at home. We named them the Fault SC (Short-Circuit Fault), EE (Earth-Earth Fault), LE (Live-Earth Fault) and LN (Live-Neutral Fault). Fault SC in Figure 1 indicates a fault that short circuits between LN, for example, due to flooding. LN shorting happens when an unearthed electrical appliance has been dipped in water or when rats chew through wires.

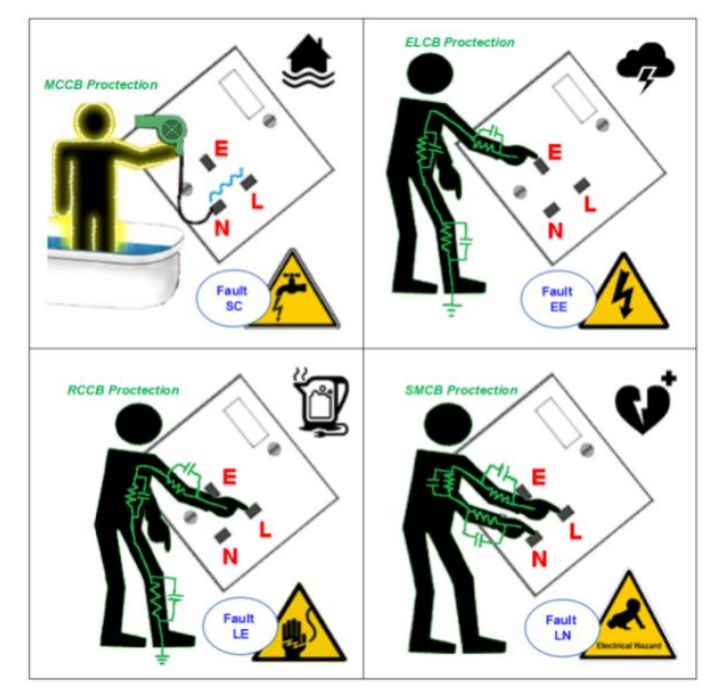

Figure1 Newly classified electrical hazard diagram

This fault is normally protected with a thermal Molded Case Circuit Breaker (MCCB) or thermal fuse devices. Fault EE indicates a fault due to lightning, during which the user could receive an electrical shock if he or she is in contact with the earth wire. This rarely happens, but if it does, the electrical source of the lightning will stop conducting for a few milliseconds, freeing the person from electrical shock. This may be the case if, for example, the person was holding an electric kettle when lightning strikes. This fault can also be protected against with a voltage sensor Earth Leakage Circuit Breaker (ELCB). However, if the earth wire is not properly connected then the ELCB will not initiate. Fault LE happens if the user accidentally touches the live wire or the appliance's live wire accidentally touches the appliance body or a metal object, resulting in the user receiving an electric shock.

Currently, the new Residual Current Circuit Breaker (RCCB) $30 \mathrm{~mA}$ is able to detect this type of fault where the residual current difference between a detected Live and Neutral wire of $30 \mathrm{~mA}$ would kick off the RCCB, protecting the user from an electric shock. According to the principles of the RCCB, if a user receives an electric shock when the appliance is working at $500 \mathrm{~mA}$, the live wire will pull the total current of $530 \mathrm{~mA}$, as illustrated in Figure 1, and leakage of $30 \mathrm{~mA}$ would pass through the human body to the ground. The Neutral wire would only return an appliance current of $500 \mathrm{~mA}$, creating a difference in the residual current of $30 \mathrm{~mA}$ and resulting in the RCCB moving to off position.

Fault LN happens when the user touches both the Live and Neutral wires at the same time. This could happen when infants, children or adults, accidentally touch the socket supply conductivity pins when the power socket was accidentally fitted half way though. This type of fault LN is the most fatal, as the current passes through the heart and stops the heartbeat. No protection device 
is currently available for this fault. Even RCCB cannot detect this type of fault as both the Live and Neutral lines have the same $30 \mathrm{~mA}$ additional current flow. However, our novel Smart Meter Circuit Breaker (SMCB) manages this type of fault.

\section{NOVEL SMCB PROTECTION DEVICE}

The new SMCB device, inbuilt in the SM, acts as a circuit breaker, too. SMs are deployed to all end-device levels of home appliances via the Internet-of-Things (IoT) for individual energy metering, and the controller can also be used for Fault LN. Our SM design is able to detect and cut off at a $30 \mathrm{~mA}$ current with a transient of one second as the threshold for the circuit breaker protection, giving time for the user to pull his or her hands back from the device after the electric shock. The SMCB resets automatically after three seconds, supplying power again to the power outlet; if the thresholds are still do not meet, the supply will be given to the appliance at a current above $30 \mathrm{~mA}$. The second rule of the SMCB is that it is programmed to detect leading Power Factor (PF) in the SM during transience of Fault $\mathrm{LN}$ at $30 \mathrm{~mA}$ current. If both rules are satisfied then the SMCB cuts off. The disadvantage of this system is that small appliances like bed lights cannot be used as they may fall into a low current mode of $30 \mathrm{~mA}$ during sleep mode. However, we feel such a sacrifice is necessary for a hazard-free electrical system in homes. The home appliances can be clustered according to load impedance, duration, functions and placement (Shashikumar et al., 2017). Figure 2 shows three types of main loads: fixed, removable and portable. The portable loads are divided into two types: colonized and alienated. The colonized loads are those already introduced in the home portable loads, and the alienated loads are newly brought or introduced into the home portable loads.

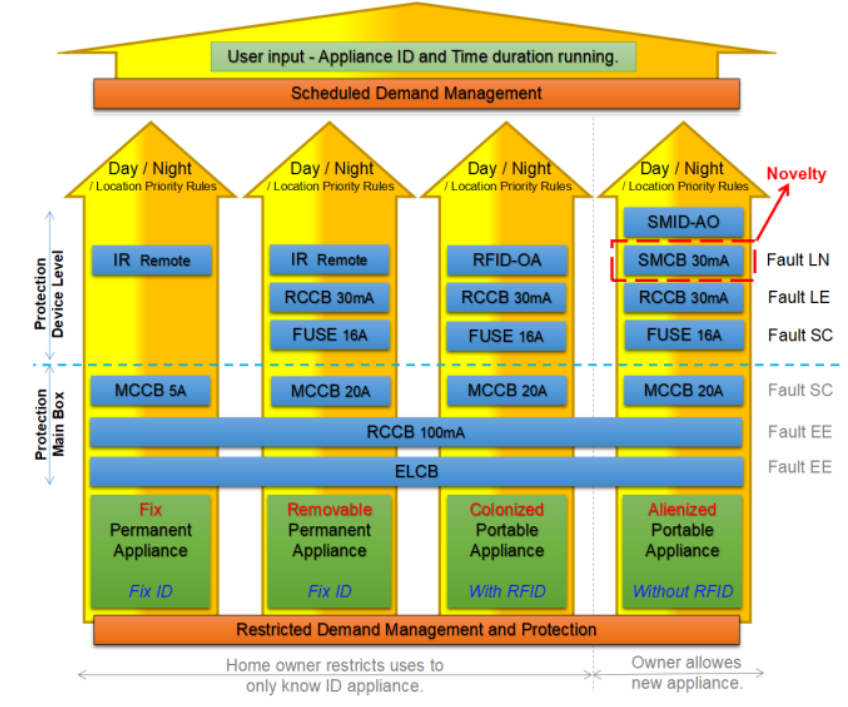

Figure 2 Flow chart illustration of demand management using classification of appliance placement, restriction and protection at Device Level (DL) and mains junction box level

\section{INNOVATIVE RFID-AO AND IR-REMOTE AS PROTECTION DEVICE}

As mentioned above, there are currently no protection devices available for Fault LN, where this fault happens at the placement of the outlet for colonized or alienated portable appliances. Boonsong and Ismail (2014) has shown how to used RFID for Wireless Monitoring of Household Electrical Power Meter. We have invented a new platform mechanism that uses RadioFrequency-Identification-Alienated-Appliance (RFID-AO) system as protection for the Fault LN for colonized portable appliances or with the existing system example SONY-RFID-AO (Sony, 2012), with our modified program. As SONY-RFID-AO is intended for the demand management 
system and we have used it as a Fault LN protection, the RFID-AO will not give an output power supply until it detects a known portable device with the Identification (ID) placed in the appliance that is plugged into the outlet.

With this arrangement, children will not easily receive any Fault LN-type shock. As for adults, Fault LN shock during plugging in an appliance could also be eliminated as our RFID-OA is programmed to activate the outlet main supply only after 10 seconds with a beep sound once it identifies the ID from the appliance. Thus, it provides ample time for the user to withdraw his or her hands from the socket. For an alienated portable appliance, the ID will not be incorporated into the appliance, as shown in Figure 2. Sockets dedicated to alienated portable appliances are equipped with our SMCB protection device, as illustrated above, which would protect users against Fault LN. For an alienated portable appliance, the RFID-AO is replaced by the SmartMeter-Identification-Alienated-Appliance (SMID-AO), which can detect a distinctive load signature needed for the demand management system as further illustrated below in this paper. RFID-OA is not used for a fixed and removable permanent appliance outlet, but a fixed SMIDAO is used to identify the appliance for the demand management system. As for Fault LN, this may not occur in a fixed and removable permanent appliance outlet without RFID-AO protection because all the appliances in this section are controlled using an Infrared-Red-Remote (IRremote). If this is not already the case, then we strongly encourage buying appliances with an IRremote as this allows the user to interact less physically with the appliance, mitigating possible electric shocks.

\section{HOW TO USE RCCB AS PROTECTION DEVICE}

As illustrated above, a RCCB $30 \mathrm{~mA}$ could be used as a shock protector for the Fault LE type. In practice, homes are not connected to RCCB $30 \mathrm{~mA}$ at the main electrical junction box, as shown in Figure 2. However, RCCB $100 \mathrm{~mA}$ or above are used as protection against Fault EE by replacing ELCB or using both in some cases. Both ELCB and RCCB $100 \mathrm{~mA}$ Fault EE-type protections work as lightning arresters. A RCCB $100 \mathrm{~mA}$ is considered very sensitive, as if lightning strikes miles away, it will easily turn off the RCCB, though the ELCB would not turn off as easily. We suggest an innovative new topology where RCCB $30 \mathrm{~mA}$ can be used at the main electrical box to protect against Fault EE and Fault LE at the same time. As shown in Figure 2, the same RCCB with different ratings can be used at the main electrical box and at the enddevice-level socket outlet of $100 \mathrm{~mA}$ and $30 \mathrm{~mA}$ to protect users from Fault EE and Fault LE, respectively. Our new architecture method mitigates sensitivity and unwanted tripping in homes with a single-phase electrical system. Fixed permanent appliances, such as ceiling fans, lights, etc., are not connected with RCCB $30 \mathrm{~mA}$ for Fault LE protection as, most of the time, these appliances are IR-remote controlled and users will not touch them, thus mitigating electric shocks. Currently, only water heater appliances must be installed with $10 \mathrm{~mA} \mathrm{RCCB}$ at Device Level (DL), and they could be further protected by installing IR-remote capabilities. Finally, Fault $\mathrm{SC}$ is protected against using a thermal fuse and MCCB protection devices ratings, respectively, at the end-device level and main electrical junction box for maximum protection.

\section{OUR SM HARDWARE}

Figure 3 shows our prototype SM, which can be enabled to perform a few tasks or the entire task at once. Our SM meter can work as an appliance energy meter, quality meter, appliance circuit breaker protection or controller (SMCB) and appliance detector via RFID (SMID-AO). It also has an optional Graphical Liquid Crystal Display (GLCD), Secure Digital (SD) card, WiFi or ZigBee data presentation system. The ZigBee coordinator, shown in Figure 3, is placed in the server or laptop, whereas the WiFi coordinator is internally built into the server. The hardware used to build our SM are a Voltage Transformer (VT) sensor, $240 \mathrm{~V}$ plug, Current Transformer 
(CT) sensor, Solid State Relay (SSR) switch, 7.5 V battery pack for back up, Arduino Uno board, PHPoc Wifi Shield board with Micro SD, Real Time Clock (RTC), CT-VT shield board, ZigBee shield board with XBee and finally a stack of GLCD4884 shield boards, as shown in Figure 3. The GLCD displays all the detected values of the appliance's RFID, SM Node-ID, Time Stamp, Date Stamp, Vrms, Irms, PF, Frequency, S, Q, P, kW/h, vTHD\%, iTHD\%, iFF and iCF.

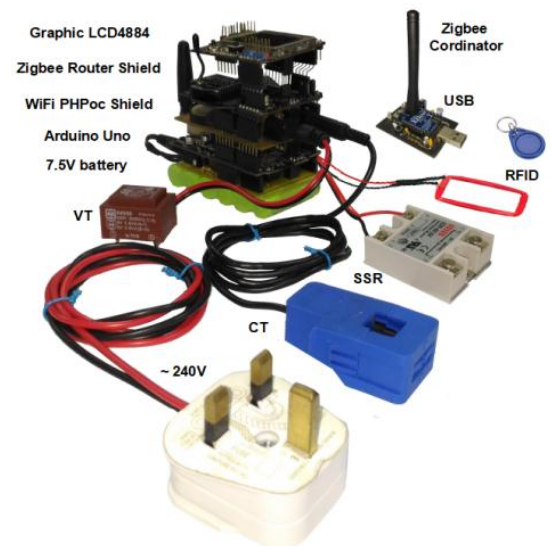

Figure 3 The hardware design of SM energy meter

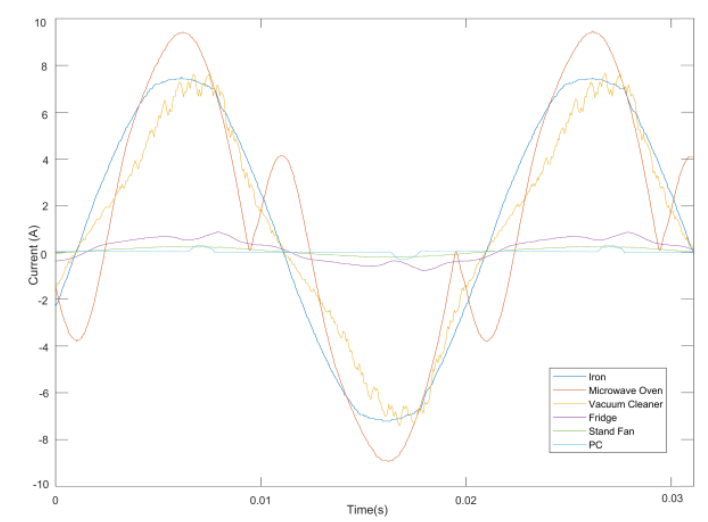

Figure 4 The instantaneous digital current waveform for six home appliances at $50 \mathrm{kHz}$ sampling

\section{SMID-OA DISTINCTIVE LOAD SIGNATURE HARDWARE RESULTS}

Figure 2 shows how a demand management system integrated with Circuit Breaker (CB) protection system which consists of appliance ID management incorporated in the SM for each type of appliance category. If the owner of the house decided to allow the occupants to use only the known colonized available appliance in the colony then our system only uses RFID-AO device for protection Fault LN and demand management system. If the owner decides to allow the occupant to use some socket outlets for their alienated portable appliances then our SMIDAO detection system is required to be activated at the socket outlet for demand management system and control. We have already published a paper (Shashikumar et al., 2016) on our design of wireless current and voltage waveform capturing logger with $50 \mathrm{kHz}$ sampling frequency. This paper design is used for our SMID-AO system. 


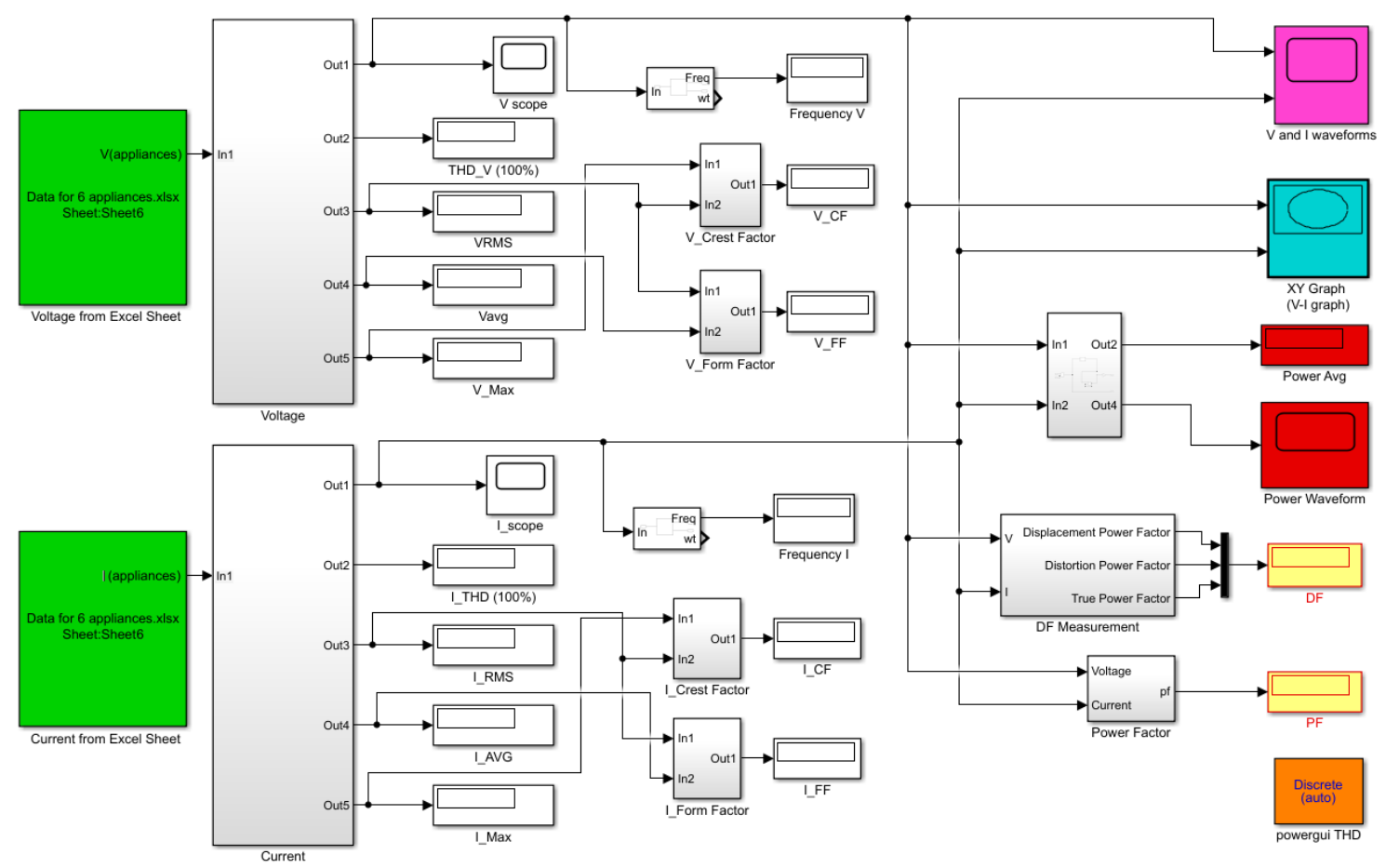

Figure 5 The instantaneous digital current and voltage are fed into the MatLAB Simulink simulation to obtain multi-metering results

Figure 3 shows the enhanced SMID-OA system with appliance-detecting capability using micro load signatures of Active Power (PQ), Current Waveform (CW), Voltage Waveform (VW), Instantaneous Admittance Waveform (IAW), Instantaneous Power Waveform (IPW), Voltage Harmonics Waveform (VH), Current Harmonics Waveform $(\mathrm{CH})$ and Switching Transient Waveform (STW) features, which are further explained by Jian Liang (2010) and Boonsong and Ismail (2014). The wireless data received by the SMID-AO server from various loads were simulated in MatLAB. The results are shown in Figure 11 in a Lissajous plot with distinctive load signature differences between six home appliance loads. They are an iron, microwave oven, vacuum cleaner, fridge, stand fan and personal computer. These differences are self-explanatory, and breakdown plots are shown in Figures 8 to 11.

To detect the appliance ID using SMID-AO, we used a V-I trajectories curve and Fryze's Power Theory, explained further by Teshome et al., (2016) and implemented in MatLAB, as shown in Figure $5 b$, where the V-I trajectories are compared with a ready data set of appliance waveforms using the correlation coefficient of waveform method. Figures 5a and 5c show MatLAB Simulink simulation data inserted using an excel spreadsheet of voltage and iron appliance currents. A plot of voltage in the time domain would be very similar throughout the home electrical system. We have only shown the Simulink data of an iron appliance, but to obtain the Figure 4 and Figure 11 data, the iron appliance Simulink block diagram Figure $5 \mathrm{c}$ is constructed in repetition.

\section{RESTRICTED DEMAND MANAGEMENT AND PROTECTION SYSTEM}

A paper by Woodhead (2018) describes the basics of a Smart City, while Wang et al. (2018) and Hoosain and Paul (2017) illustrate how the demand management system can be used at the end consumer demand side and also at the grid side. Susanto et al. (2018) state how simple intelligent management of a refrigerator thermostat could save energy. Figure 2 shows the architecture flow of our novel Restricted Demand Management System incorporated with a protection system. Our system acts as a first line of defense for intelligent demand management before being fed into the commonly implemented scheduled demand management system to achieve a flattened base load 
line in a day in a single-phase home electrical system. We have chosen a simple studio apartment home layout, as shown in Figure 6, with Device Level Load Monitoring (DLLM) and Device Level Load Protection (DLLP) placed in strategic places. In some locations, SM or protection devices are not needed due to the type of appliance classification, as illustrated in Figure 2. Some SM also do not need SM-RFID or SMID-AO because they are equipped with fixed ID in the SM, like, for example, the fixed load like a ceiling fan. Some socket outlets need SMID-AO to find the portable appliance ID plug in the socket outlet due to the owner's preference of allowing alienated appliances to be used in the home.

Colonized portable appliances need only SM-RFID and not SMID-AO because the appliances are all fixed with RFID transmitters, as shown in the storeroom section of Figure 6. Also, our system prevents users from plugging portable appliances into sockets that are overloaded, as highlighted in red in Figure 6.

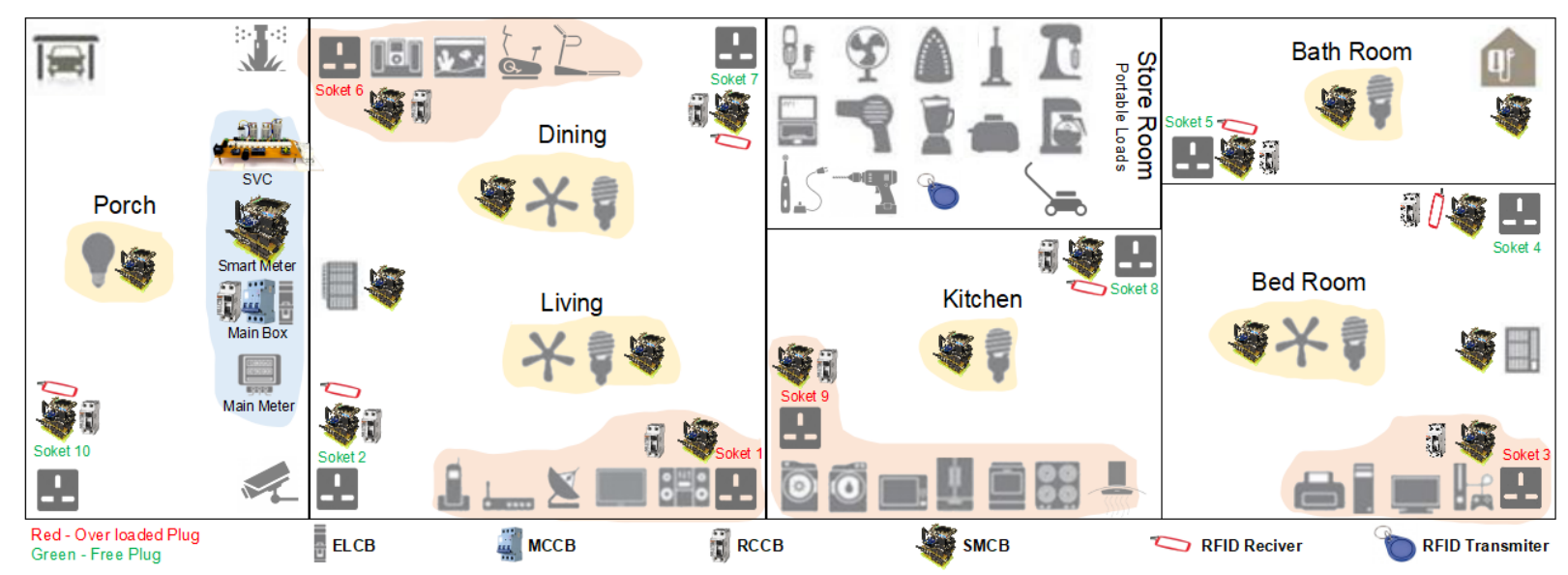

Figure 6 A home topology layout of appliances model fitted with SM

Our restricted demand management system will only allow the green highlighted outlets to be used by the portable appliance, as they are free from overloads. All house owners prioritize utility protection from key hazards to make sure the occupants of their house or building are safe. Our Restrictive Demand Management and Protection system provides both protection and utility bill reduction at the same time. Furthermore, the system employs a simple, strict rule of if-else priority programming design for restrictions in the server that are connected wirelessly via ZigBee to the DLLM SM modules. Figure 7a shows our priority list design table for the Figure 6 home electrical layout system with a base load line appliance of a continuous load, for example, a Closed-circuit Television (CCTV), modem and security alarm that works twenty-four hours a day. Next, the daytime and nighttime sections are set as priority level 1. The comfort mode level 1 is set to high, as shown in Figure 7a, which includes the lights, fan and air-conditioner. These appliances are also a high priority as an occupant needs to be comfortable in a home with heating or air conditioning, depending on the weather. Next, cooking is set as priority level 2, since preparing meals is a necessity for the occupant of a home. Entertainment is set as priority level 3, which includes electronic appliances such as a television, radio and desktop computer. The level 4 priority setting has a day and night appliance selection; this shifting mode allows a particular appliance to work only at certain times of the day. The style shifting mode enables the user to shift their normal ways of conducting their work or chores at home, resulting in cost savings in utility usage. 


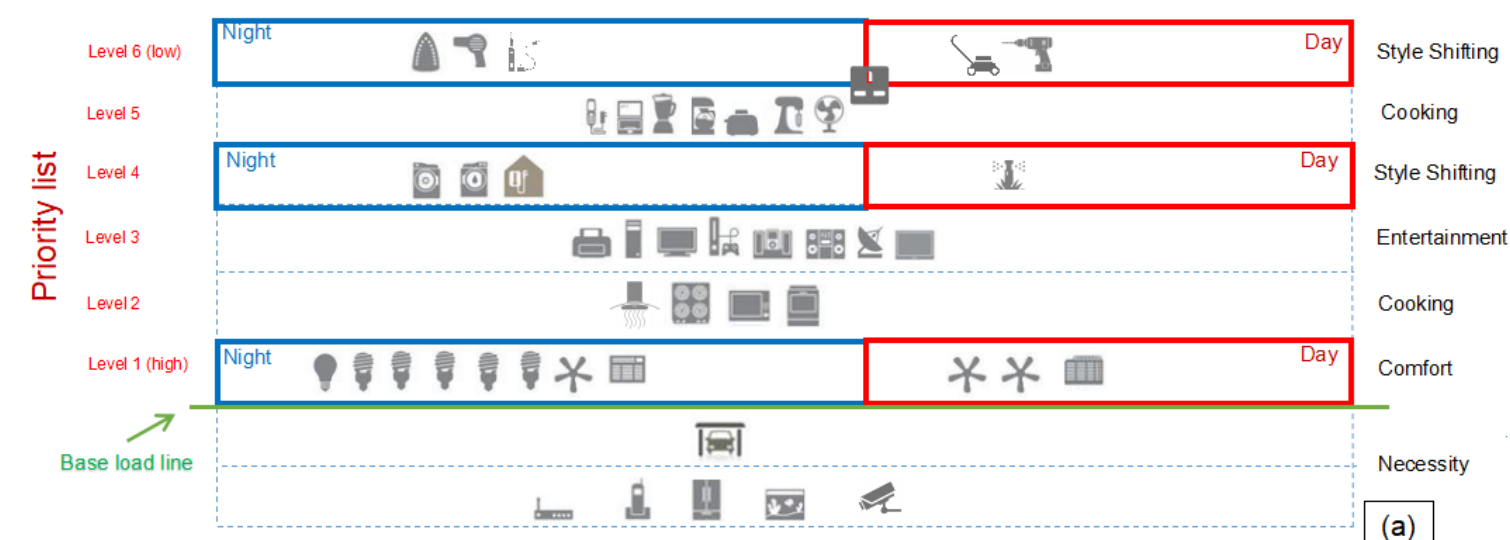

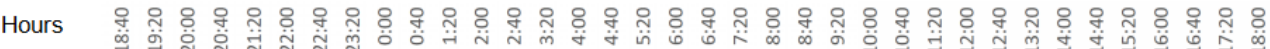
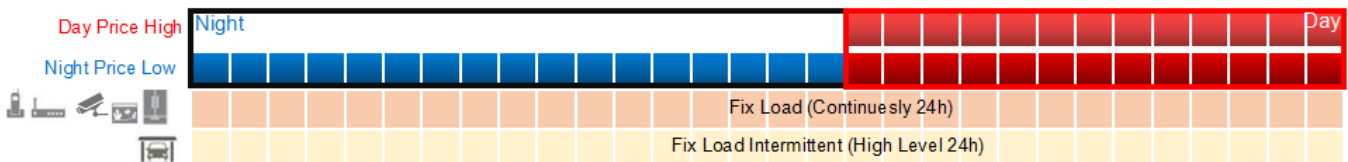

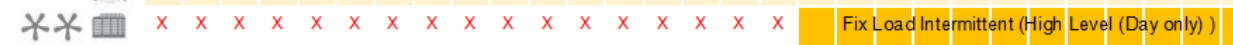
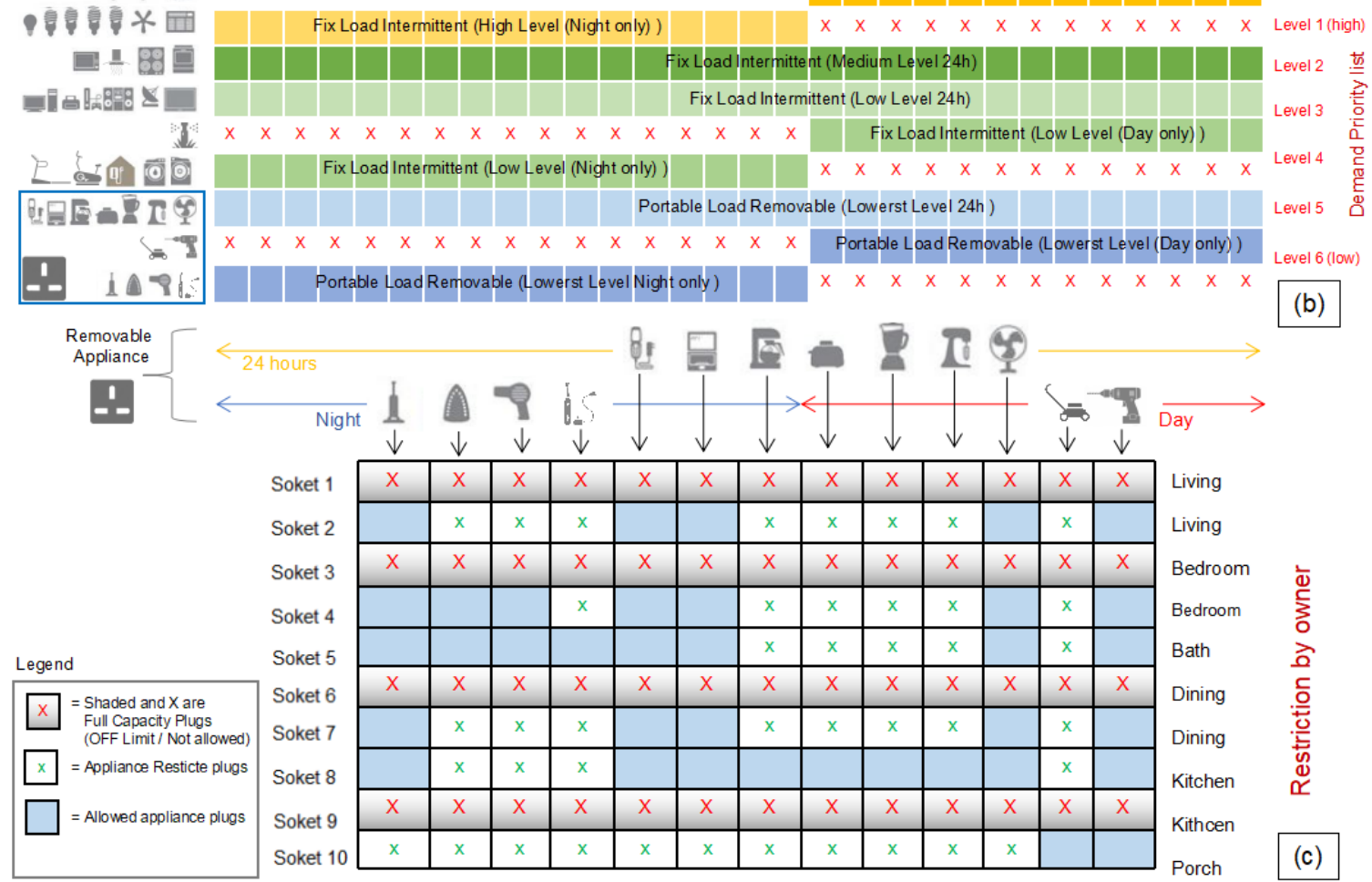

Figure 7 Home utility table structured for Restricted Demand Management System

The washing machine is an example of style shifting that is programmed to be used at night only due to the cost of electricity, which is cheaper at night than the day, as shown in Figure $7 \mathrm{~b}$. Similarly, there is also another set of appliances grouped at level 6, as the lowest priority, with day and night selection. The second lowest priority (level 5) is also used for appliances used for light cooking, as shown in Figure 7a. Both the table designs in Figures $7 \mathrm{a}$ and $7 \mathrm{~b}$ are converted to if-else statements codes in our novel Restricted Energy Demand Management System server for the home layout as shown in Figure 6. 

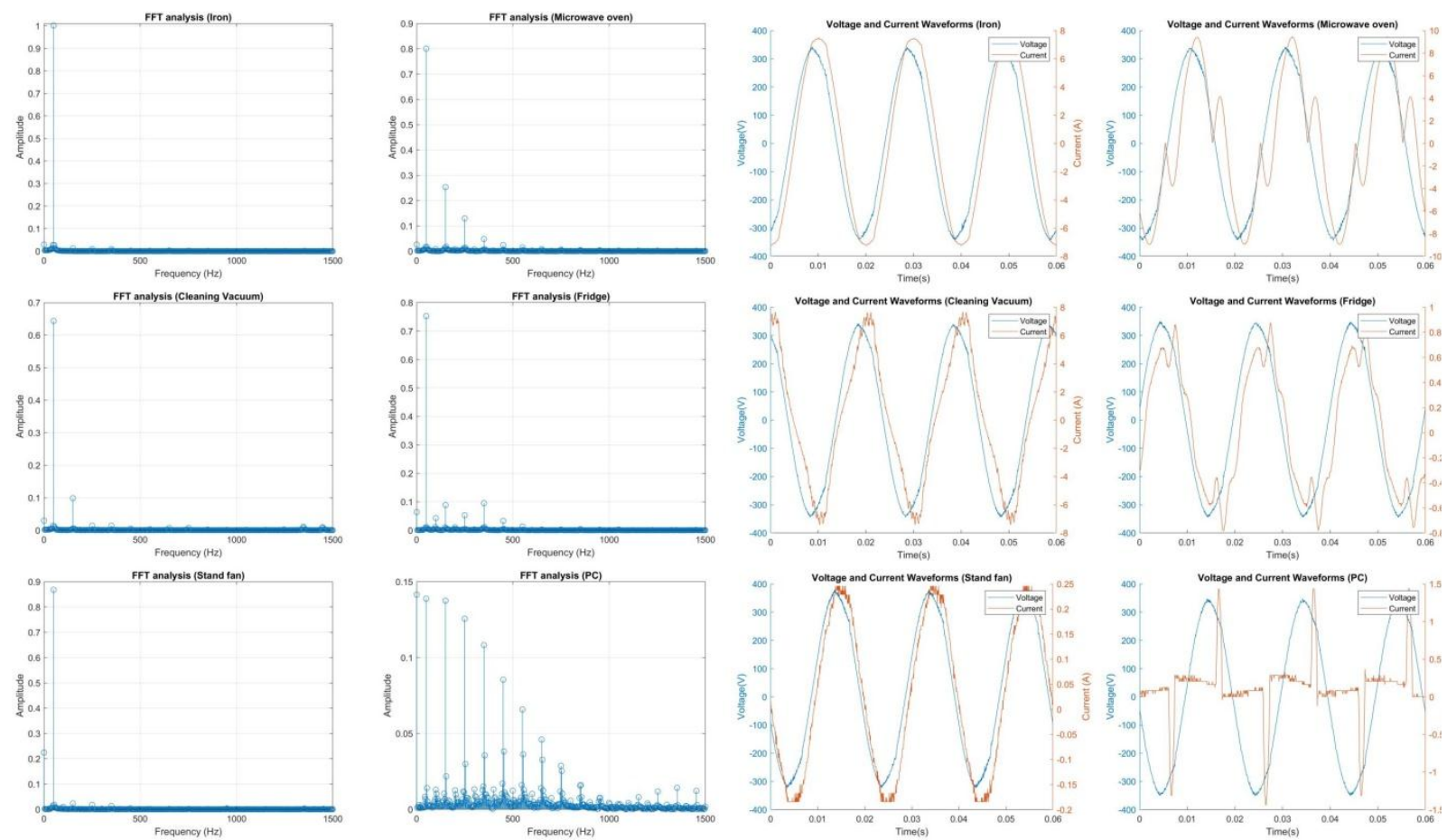

Figure 8 FFT-iTHD analysis for six appliances

Figure $9 \mathrm{VW}$ and $\mathrm{CW}$ analysis for six appliances
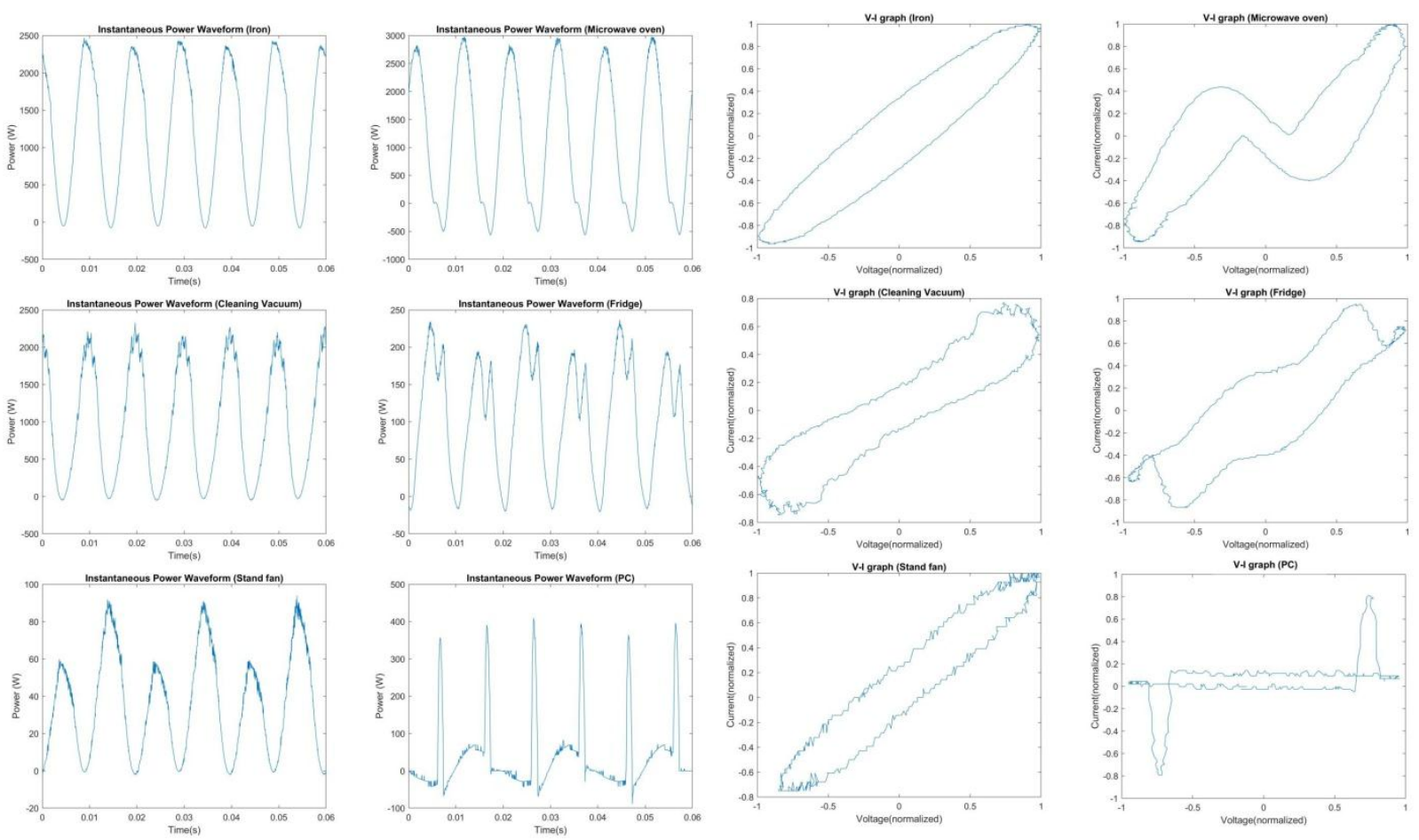

Figure 10 IPW analysis for six home appliances
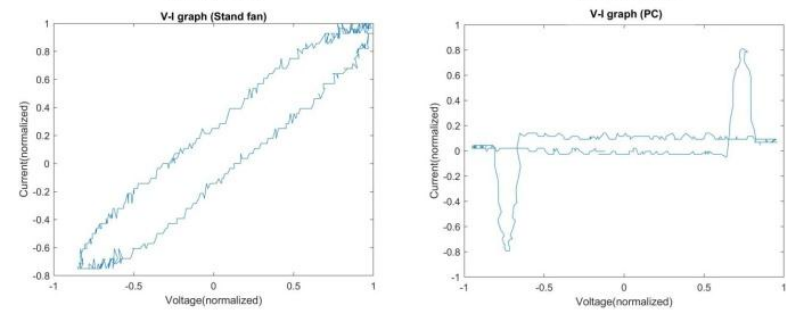

Figure 11 V-I Trajectory analysis for six appliances

To further optimize our Restricted Demand Management System, the commonly used Scheduled Demand Management System is then implemented in the style shifting priority level 4 and 5 in Figure 7a. For the Scheduled Demand Management System, the user inputs the appliance ID and time duration of an appliance as shown in Figure 2. Finally, our Restricted Demand Management System is also incorporated into a DL protection system with the restriction for each socket outlet designed by the home owner. An example of the DL protection system restrictions is indicated 
in Figure 7c for the home layout in Figure 6. The restriction of a socket outlet protects the appliances. For example, we would not want a cooking appliance to be used in a bedroom area socket outlet due to fire hazards. An SM connected to each socket outlet identifies the connected appliance, and a restriction is imposed according to our design in Figure 7c. The table in Figure $7 \mathrm{c}$ is used as a guideline. Some socket outlets are restricted for all appliances, as they already have overload appliances connected to them, whereas, for others, only limited appliances are restricted.

\section{CONCLUSION}

Currently, available home electrical system architecture does not guarantee shock protection or emphasizes the principles of electrical safety fully. Even-though there is already available some safety equipment in the market it is not used fully utilized by consumers to protect themself from electrical hazards due to reasons of inadequate knowledge or reluctance to spend more money. One type of protection is the use of a $30 \mathrm{~mA}$ RCCB in a DLLP architecture system, as we propose in this paper. At the moment, water heaters are the only devices that use this mode of architecture. The $30 \mathrm{~mA}$ RCCB at DL can prevent Fault LE shock, but for Fault LN shock there are no CB protection devices available.

To protect against Fault LN shock, we suggest two methods. The first is to use our SMCB where an SM can detect any $30 \mathrm{~mA}$ fault, though the disadvantage is that any appliance working below $30 \mathrm{~mA}$ cannot be used in the device-level appliance outlet. The second method is to use the existing SONY-RFID-AO demand management system, which can easily be modified and programmed to become a protection system against Fault LN shock, as we have suggested in this paper. We also suggest using our own SMID-AO system, which can detect the appliance ID without the use of RFID and allows any type of appliance, whether colonized or alienated. However, this system is more expensive than our SMCBs. Finally, we have also suggested using a novel Restrictive Demand Management System, which is simpler and could be incorporated into a commonly used Scheduled Demand Management System to reduce utility bills in homes. The disadvantage of this system is that each home with a different layout will require a new priority list table designed for it.

\section{REFERENCES}

Alrawi, A.H., Moghavvimi, M., Ibrahim, W., 2010. Novel Idea to Monitor and Measure Blood Hemoglobin Noninvasively. African Journal of Biotechnology, Volume 9(54), pp. 92959306

Boonsong, W., Ismail, W., 2014. Wireless Monitoring of Household Electrical Power Meter using Embedded RFID with Wireless Sensor Network Platform. International Journal of Distributed Sensor Network, Volume 10(6), pp. 1-10

Hoosain, M.S., Paul, B.S., 2017. Smart home: A Domestic Demand Response and Demand Side Energy Management System for Future Smart Grids. International Conference on the Domestic Use of Energy (IEEE), pp. 1-5

Jian Liang, Sion K.K. Ng, Gail Kendail, John W.M. Cheng, 2010. Load Signature Study Part I: Basic Concept, Structure, and Methodology. Transactions on Power Delivery on IEEE, Volume 25(2), pp. 551-560

Shashikumar, K., Venkataseshaiah, C., Sim, K.S., 2016. Juggling an Arduino for Multi-meter, Load Profiling and Novel Waveform Capture Logger Application. In: The $12^{\text {th }}$ International Colloquium on Signal Processing \& its Applications (CSPA) IEEE, pp. 119-123

Shashikumar, K., Venkataseshaiah, C., Sim, K.S., 2017. Automatic Sequential Reactive Power Compensation and Harmonic Suppression at Loads using Appliance Clustering and Power 
Quality Monitoring. In: International Conference on Robotics, Automation and Sciences (ICORAS) IEEE, pp. 1-6

Sony Press Release, 2012. Sony Develops "Authentication Outlet" Where Electricity Use can be Managed and Consumed on a Per-User and Per-Device Basis. Available Online at https://www.sony.net/SonyInfo/News/Press/201202/12-023E/, Accessed on 14 February 2012

Susanto, E., Idrus, M., Nasruddin, N., Budihardjo, B., 2018. An Experimental Investigation into the Effect of Thermostat Setting on the Energy Consumption of Household Refrigerators. International Journal of Technology, Volume 9(2), pp. 364-371

Teshome, D.F., Huang T.D., Lian, K.L., 2016. Distinctive Load Feature Extraction based on Fryze's Time-domain Power Theory. Power and Energy Technology Systems Journal on IEEE, Volume 3(2), pp. 60-70

Wang, K., Li, H., Maharjan, S., Zhang, Y., Guo, S., 2018. Energy Scheduling for Demand Side Management in the Grid. Transaction on Green Communications and Networking on IEEE, Volume 2(2), pp. 596-611

Woodhead, R., 2018. Building a Smarter City. International Journal of Technology, Volume 9(7), pp. 1509-1517 